\title{
Multiple Model Unscented Kalman Filtering in Dynamic Bayesian Networks for Intention Estimation and Trajectory Prediction
}

\author{
Jens Schulz ${ }^{1}$, Constantin Hubmann ${ }^{1}$, Julian Löchner ${ }^{1}$, and Darius Burschka ${ }^{2}$
}

\begin{abstract}
Dynamic Bayesian networks (DBNs) are a popular method for driver intention estimation and trajectory prediction. To account for hybrid state spaces and non-linear system dynamics, sequential Monte Carlo (SMC) methods are often the inference method of choice. However, in state estimation problems with high uncertainty, SMC methods typically suffer from either high complexity (using many samples) or low accuracy (using an insufficient number of samples). In this paper, we present a multiple model unscented Kalman filter based DBN inference method for driver intention estimation and multi-agent trajectory prediction. This inference method reduces complexity, while still keeping the benefits of samplebased evaluation of non-linear and non-continuous transition models. Firstly, the state of the DBN is approximated as a mixture of Gaussians and estimated over time by tracking the multi-agent system. Secondly, a probabilistic forward simulation of the belief is performed to generate interaction-aware trajectories for all agents and all intention hypotheses. The proposed method is compared to SMC-based inference methods in terms of accuracy, variance and runtime in both simulations and real-world scenarios.
\end{abstract}

\section{INTRODUCTION}

To drive in an anticipatory and cooperative manner, autonomous vehicles need to anticipate the future behavior of surrounding traffic participants and estimate their current intentions. The trajectories of multiple vehicles in a traffic scene are often highly interdependent because drivers must avoid collisions, comply with traffic rules, and thus react to other drivers' actions. This introduces the need for combinatorial and interaction-aware motion prediction, which still represents a great challenge today [1].

Dynamic Bayesian networks (DBNs) are commonly used for driver intention estimation because they allow the definition of domain specific hierarchies within the decision making process of human drivers and the modeling of interdependencies between multiple agents. In our previous work [2], we proposed such a framework which applies sequential Monte Carlo (SMC) inference to estimate intentions and predict interaction-aware trajectories for all vehicles in a scene. SMC methods have the advantage that they can represent arbitrary distributions (hybrid, non-Gaussian, multi-modal) and can be applied to highly non-linear systems. However, in state estimation problems with high uncertainty, e.g., as a result of unknown intentions, varying human behavior and noisy measurements, they typically suffer from either high complexity or low accuracy, depending on the number

\footnotetext{
${ }^{1}$ Jens Schulz, Constantin Hubmann, and Julian Löchner are with BMW Group, Munich, Germany \{jens.schulz | constantin.hubmann | julian.loechner\}@bmw.de

${ }^{2}$ Darius Burschka is with the Department of Computer Science, Technical University of Munich, Germany burschka@tum.de (c) 2018 IEEE
}

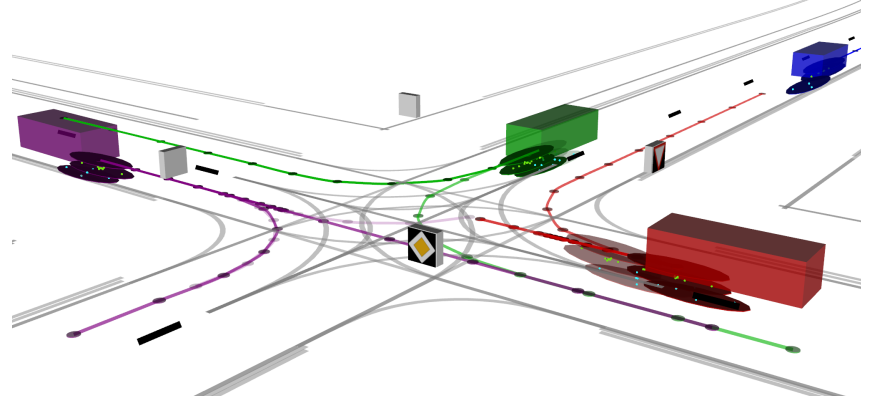

Fig. 1. Estimated belief represented by a mixture of Gaussians and corresponding sigma points and interaction-aware trajectory prediction for the different possible intentions.

of samples used. Although using fewer samples does not introduce bias, the variance in the results increases due to the randomness in the sampling process. Furthermore, the problems of particle degeneracy (just a few particles represent the state well) and particle impoverishment (most particles have the same value) arise because of imprecise models and importance resampling [3].

In this paper, we propose a multiple model unscented Kalman filter (MM-UKF) based inference method for our previously presented intention estimation and trajectory prediction framework. As MM-UKF approximates the belief using a mixture of Gaussians, it is not associated with the aforementioned problems of SMC methods, while still keeping the benefits of sample-based evaluation of complex, non-linear and non-continuous transition models.

The presented DBN models the development of a traffic situation as a stochastic process comprising multiple interacting agents. The continuous actions of each agent are conditioned on the current environment and the agent's route and maneuver intentions (see IV-A for definitions). To account for interdependencies between multiple agents, each of the discrete modes of the MM-UKF represents one of the possible combinations of discrete intentions of all agents, whereas the continuous states are represented by one multivariate Gaussian given each mode. A typical scenario is depicted in Fig. 1, showing the Gaussian mixture distribution and the corresponding sigma points.

The proposed inference method is evaluated in both simulations and real world scenarios and compared to the previously presented SMC inference. The results show that given the discrete intentions of all agents, the state can reasonably be approximated by a single multivariate Gaussian. MMUKF achieves lower variance and higher accuracy compared to SMC inference with a similar runtime. 


\section{RELATED WORK}

\section{A. Intention Estimation and Motion Prediction}

Intention estimation and motion prediction of human traffic participants have been widely studied within the field of autonomous vehicles. Commonly, route and maneuver intentions are estimated with either discriminative classifiers, such as support vector machines (SVMs) [4], random forests (RFs) [5], or artificial neural networks (ANNs) [6], or with generative models, such as Bayesian networks [7]. The prediction of continuous trajectories is often based on regression methods such as Gaussian processes (GPs) [8], [9], RFs [10], ANNs [11] or planning-based methods [12].

Modeling the development of a traffic situation as a stochastic process conditioned on hidden variables representing the agents' intentions allows both inferring these intentions at the current time by incorporating measurements, and predicting the future by iteratively applying the transition models to the estimated belief (forward simulation). Therefore, the problems of intention estimation and motion prediction are handled within a combined framework, utilizing the same behavior models. In [8], multiple GPs are defined conditioned on the maneuver intention of a driver (turn-left, turn-right, go-straight, stop-and-go), allowing the estimation of his current intention given a set of observations. These GPs are then used as transition models of a particle filter in order to predict the continuous trajectory. As contextual information, such as the existence of surrounding traffic participants, is not incorporated, the framework is not suited for multi-agent scenarios. In our previous work [12], we address the interrelated problems of behavior generation of the ego vehicle and behavior prediction of the surrounding vehicles in a combined manner. Multi-agent maneuvers based on the concept of homotopy are determined and corresponding trajectories are optimized using mixed integer quadratic programming. A multiple model Kalman filter that uses the trajectories as a transition model infers the intentions of surrounding agents. The ego vehicle is then controlled so that it complies with the most probable prediction. In [10], a framework is presented that describes the traffic scene using a DBN consisting of multiple interacting agents. Learned context-dependent action models are conditioned on the route intention, allowing a reduction in learning complexity. Using SMC inference, they are able to estimate the multi-modal hybrid belief state and predict the future scene development based on a non-linear model. Although they do not evaluate the route estimation capabilities, they show that their trajectory prediction outperforms a constant turn rate and velocity (CTRV) model in simulation. In our previous work [2], we also model the development of a traffic scene with a DBN consisting of multiple interacting agents using SMC inference. The decision making process of each traffic participant is divided into three levels: route intention, maneuver intention and action (see Sec. IV-A for definitions). Possible routes and maneuvers are queried at runtime given a digital map, and interdependencies between agents as well as dependencies on the static context (e.g., road curvature) are represented within the network structure. By including all agents within the state space, it is possible to explicitly account for the combinatorial aspect of interacting agents. We show that our interaction-aware approach outperforms pure map-based and CTRV models that neglect other participants.

As this paper focuses on comparing MM-UKF-based inference to our previous SMC inference, we refer to [2] for a more detailed review of behavior prediction literature.

\section{B. Inference in hybrid dynamic Bayesian networks}

DBNs are probabilistic graphical models that consist of multiple random variables which are connected via directed arcs representing their dependencies. Each variable is described by a probability distribution conditioned on its parents. A detailed overview of different possible inference algorithms for specific variants of DBNs is presented in [13].

In the special case of discrete-only hidden variables or when transition and observation models are conjugate (e.g., linear-Gaussian), exact inference is generally possible. However, for non-linear DBNs with hybrid state space (i.e., both discrete and continuous hidden variables), such as presented within this work, approximate inference becomes necessary. A common method for inference in arbitrary DBNs are SMC methods, as they can represent arbitrary distributions and cope with non-linear system dynamics. SMC has already been successfully applied for DBN inference in the context of behavior prediction, e.g., by [10] and in our previous work [2]. However, particle-based inference often suffers from high complexity, as many particles may be needed to approximate the belief appropriately and to reduce the variance caused by randomness in the sampling process. Another trade-off can be found in the problems of degeneracy and impoverishment: because of inaccurate models and system noise, most particles will end up with small weights eventually (because they represent the state poorly) and only few will have non-negligible weight. A method to reduce this degeneracy and focus the set of particles to regions in the state space of high probability is called importance resampling, which, however, introduces another problem called impoverishment, meaning that most of the particles represent exactly the same values. A detailed analysis of these problems can be found in [3].

In the special case of a switching state space model, it is possible to represent the belief by a mixture of Gaussians and apply an (interacting) multiple model extended or unscented Kalman filter. Then, the discrete variables are represented by the mode on which the underlying EKFs/e UKFs are conditioned on. The unscented Kalman filter has often been shown to be superior to the extended Kalman filter (e.g., [14], [15], [16]). Furthermore, the UKF does not require the analytic evaluation of any derivatives, making it simpler and more widely applicable than the EKF [13]. A comparison of EKF, UKF, and SMC was conducted by [16], highlighting the benefits of UKF-based inference. They employ a twostep serial process by first sampling the discrete variables and then applying EKF or UKF for the remaining variables for each particle (which they refer to as 'non-strict' Rao- 
Blackwellization). In [17], an in-depth analysis of UKF was conducted, demonstrating that the advantages and disadvantages of UKF and SMC in terms of complexity and accuracy depend on the dimensionality of the state space and the problem at hand. Furthermore, the authors recommend selection of sigma points that are not too close to the mean because otherwise nonlinear effects away from the mean are not accounted for, although this may be required by the actual spread of the distribution.

\section{Problem Statement}

A traffic scene $S$ consists of a set of agents $\mathcal{V}=\left\{V^{0}, \cdots, V^{K}\right\}$, with $K \in \mathbb{N}_{0}$, in a static environment (map) with discrete time, continuous state, and continuous action space. The map consists of a road network with topological, geometric and infrastructure (yield lines, traffic signs, etc.) information as well as the prevailing traffic rules. At time step $t$, the set of agents $\mathcal{V}$ is represented by their kinematic states $X_{t}=\left[\boldsymbol{x}_{t}^{0}, \cdots, \boldsymbol{x}_{t}^{K}\right]^{\top}$, route intentions $R_{t}=\left[r_{t}^{0}, \cdots, r_{t}^{K}\right]^{\top}$, and maneuver intentions $M_{t}=\left[m_{t}^{0}, \cdots, m_{t}^{K}\right]^{\top}$. The kinematic state $\boldsymbol{x}_{t}^{i}=\left[x_{t}^{i}, y_{t}^{i}, \theta_{t}^{i}, v_{t}^{i}\right]^{\top}$ of agent $V^{i}$ consists of the Cartesian position, heading, and absolute velocity. The agent's length and width are considered to be given deterministically by the most recent measurement and, for the sake of brevity, are not included within $\boldsymbol{x}^{i}$. The route intention $r_{t}^{i}$ defines a path through the road network the agent desires to follow, the maneuver intention $m_{t}^{i}$ the desired order relative to other agents in cases of intersecting or merging routes (see Sec. IV-A for detailed definitions). Other types of maneuvers such as lane changes or overtaking are not considered within this work. At each time step, each agent executes an action $\boldsymbol{a}_{t}^{i}$ that depends on its intentions, the map and the kinematic states of all agents, transforming the current kinematic state $\boldsymbol{x}_{t}^{i}$ to a new state $\boldsymbol{x}_{t+1}^{i}$. The actions of all agents are denoted as $A=\left[\boldsymbol{a}_{t}^{0}, \cdots, \boldsymbol{a}_{t}^{K}\right]^{\top}$. The complete dynamic part of a scene is thus described by $S_{t}=\left[X_{t}, R_{t}, M_{t}, A_{t}\right]^{\top}$. At each time step, a noisy measurement $Z_{t}=\left[\boldsymbol{z}_{t}^{0}, \cdots, \boldsymbol{z}_{t}^{K}\right]^{\top}$ with $\boldsymbol{z}_{t}^{i}=\left[z_{x, t}^{i}, z_{y, t}^{i}, z_{\theta, t}^{i}, z_{v, t}^{i}\right]^{\top}$ is observed according to the distribution $p\left(Z_{t} \mid X_{t}\right)$, containing information about the kinematic states of all agents. The number of agents, possible routes and maneuvers is arbitrary and may change over time: agents may appear or disappear and their possible routes and maneuvers need to be adapted accordingly.

The objective is to estimate the route and maneuver intentions $(R, M)$ of all agents at the current time. This work focuses on an alternative inference method with improved runtime complexity compared to our previous approach [2] while keeping high estimation accuracy.

\section{APPROACH}

We describe the development of a traffic scene as a Markov process consisting of all agents in a scene. Modeling this process in a DBN allows explicit specification of relations between agents, the definition of causal as well as temporal dependencies and handling of the uncertainty of measurements and human behavior. The decision making process

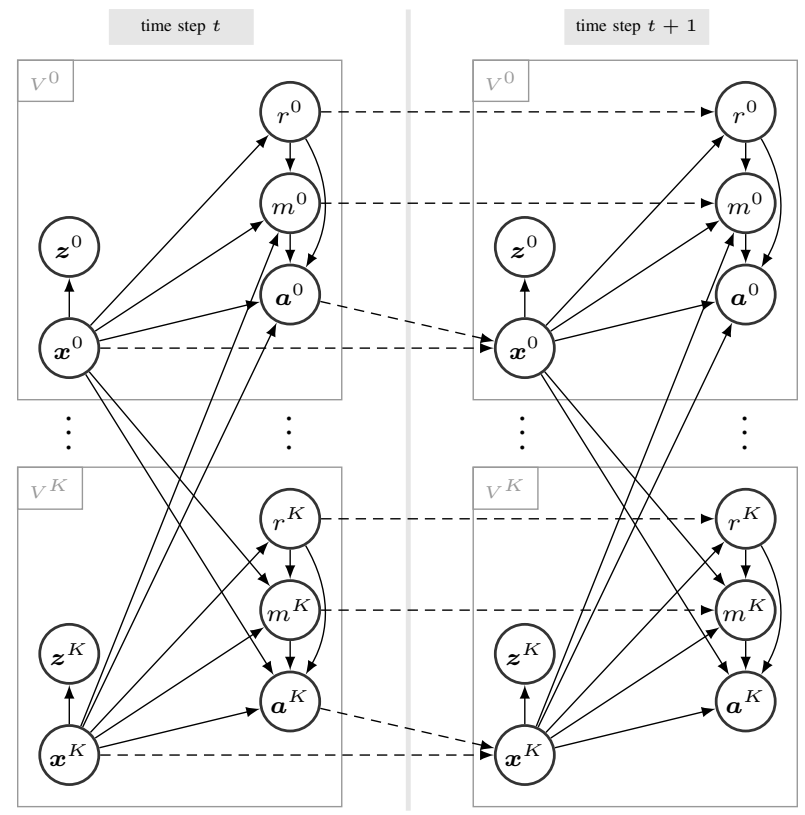

Fig. 2. dynamic Bayesian network (DBN) showing the interdependencies between agents $V^{i}$. Random variables are drawn as circles, causal and temporal dependencies as solid and dashed arrows, respectively. $r, m$, and $a$ depend on the map. [2]

of each agent is composed of three hierarchical layers: the route intention, i.e. the path the agent desires to follow, the maneuver intention, i.e. whether it is going to pass a conflict area at an intersection before or after another agent, and the continuous action comprising acceleration and yaw-rate. First, the set of possible routes and maneuvers is determined given a topological map. Each agent's continuous action is then derived by context-dependent behavior models given its route and maneuver intentions. Recurrently updating the belief of the DBN with observations of the agents' poses and velocities allows the drivers' intentions to be inferred. A probabilistic trajectory prediction is generated by forward simulation of the current belief. Thus, the predicted trajectories explicitly incorporate the intentions of drivers and respect the interdependencies between multiple agents.

Fig. 2 shows two consecutive time slices of the DBN and the dependencies between the single random variables. In general, this DBN describes a hybrid, non-linear system with a multi-modal, non-Gaussian belief. However, in contrast to our previous work [2], we present multiple model unscented Kalman filtering for inference, which approximates the belief with a mixture of Gaussians. To account for changing situations, the network structure is adapted at runtime (creating and deleting agents as well as route/maneuver hypotheses). Thus, it can be applied to varying situations with an arbitrary number of agents, intention hypotheses and different road layouts.

\section{A. Transition Models}

This section gives an overview of the transition models of the DBN. As the focus of this work is the comparison of the MM-UKF inference to the previously presented SMC 

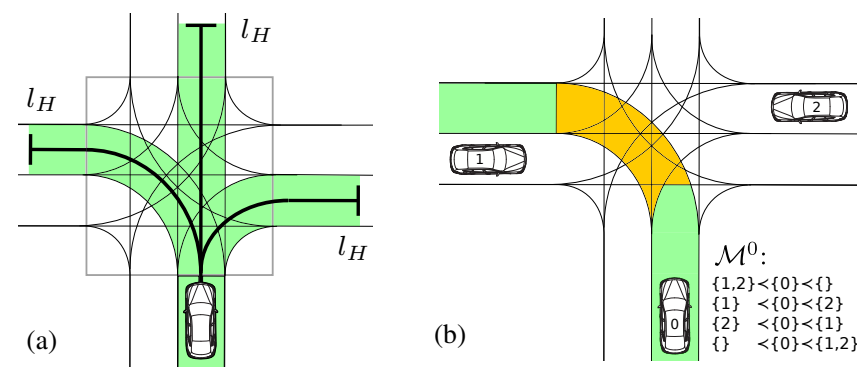

Fig. 3. (a): Possible routes (green). (b): Conflict areas (yellow) from $V^{0}$ 's perspective for turning left and the resulting four possible maneuvers, representing the sequence of agents passing the conflict areas. [2]

inference, we refer to our previous work [2] for a more detailed description of the transition models used. For the sake of brevity, the superscript denoting the single agent is omitted in this subsection (e.g., $\boldsymbol{x}$ instead of $\boldsymbol{x}^{i}$ ).

1) Vehicle Kinematics: the action $\boldsymbol{a}=[a, \dot{\theta}]^{\top}$ of an agent comprises the longitudinal acceleration $a$ and the yaw rate $\dot{\theta}$. It is the outcome of the agent's decision making process (described later in this section) and is conditioned on the current context and the agent's intentions. The kinematic state is predicted according to the probability distribution $p\left(\boldsymbol{x}_{t+1} \mid \boldsymbol{x}_{t}, \boldsymbol{a}\right)=\mathcal{N}\left(\hat{\boldsymbol{x}}_{t+1}, \boldsymbol{Q}\right)$, with

$$
\hat{\boldsymbol{x}}_{t+1}=\left(\begin{array}{c}
x_{t}+v_{t} \Delta T \cos \left(\theta_{t+1}\right)+\frac{1}{2} a_{t} \Delta T^{2} \cos \left(\theta_{t+1}\right) \\
y_{t}+v_{t} \Delta T \sin \left(\theta_{t+1}\right)+\frac{1}{2} a_{t} \Delta T^{2} \sin \left(\theta_{t+1}\right) \\
\theta_{t}+\dot{\theta_{t}} \Delta T \\
v_{t}+a_{t} \Delta T
\end{array}\right)
$$

and $\boldsymbol{Q}=\operatorname{diag}\left(\sigma_{x}^{2}, \sigma_{y}^{2}, \sigma_{\theta}^{2}, \sigma_{v}^{2}\right)$. Although this model is simplistic, we argue that it is sufficient for prediction purposes.

2) Measurement: high-level cuboid objects representing the single agents in a scene are used as measurements, thus low-level sensor specifics are abstracted. The data association, i.e., object detection and tracking, is considered to be given as it is handled by a different software module. We model the kinematic state of each agent to be measured with zero-mean Gaussian noise, such that each measurement $\boldsymbol{z}=\left[x_{z}, y_{z}, \theta_{z}, v_{z}\right]^{\top}$ is distributed according to $p(\boldsymbol{z} \mid \boldsymbol{x})=$ $\mathcal{N}(\hat{\boldsymbol{z}}, \boldsymbol{R})$, with $\hat{\boldsymbol{z}}=\boldsymbol{x}$ and $\boldsymbol{R}=\operatorname{diag}\left(\sigma_{z_{x}}^{2}, \sigma_{z_{y}}^{2}, \sigma_{z_{\theta}}^{2}, \sigma_{z_{v}}^{2}\right)$.

3) Route Intention: the desired route $r \in \mathcal{R}$ of an agent represents the first layer of its decision making process. It is given by a sequence of consecutive lanes and serves as a path that guides the agent's behavior. The set of possible routes $\mathcal{R}$ is determined given the agent's pose, the topological map, and a specified metric horizon $l_{H}$ (see Fig. 3a). Initially, the desired route $r$ is considered to be distributed uniformly across the set of possible routes: $p\left(r_{i} \mid \boldsymbol{x}, \mathrm{map}\right)=|\mathcal{R}|^{-1}, \quad \forall r_{i} \in \mathcal{R}$. For the sake of problem simplification, the decision on a route is considered constant, i.e., the probability of a route switch is assumed to be zero.

4) Maneuver Intention: the desired maneuver $m \in \mathcal{M}$ represents the second layer of the decision making process and defines the sequence, in which agents desire to merge or cross at conflict areas (where merging or intersecting lanes overlap), as shown in Fig. 3b. For all vehicle pairs $\left\langle V^{i}, V^{j}\right\rangle$ having a common conflict area, the maneuver of vehicle $V^{i}$ specifies, whether it will pass this conflict area before $\left(V^{i} \prec V^{j}\right)$ or after $V^{j}\left(V^{i} \succ V^{j}\right)$. This kind of maneuver is based on the concept of pseudo-homotopy of trajectories, which we developed in an earlier work [12]. Our data suggests, that vehicles that have right of way are typically only insignificantly influenced by other vehicles approaching the intersection. Thus, different maneuvers are only considered for vehicles that do not have right of way.

An agent's set of possible maneuvers $\mathcal{M}$ is derived given its route, the map, and the kinematic states of all agents. The desired maneuver $m$ is initially distributed uniformly across the set of possible maneuvers: $p\left(m_{i} \mid X, r\right.$, map $)=|\mathcal{M}|^{-1}$, $\forall m_{i} \in \mathcal{M}$. The decision on a maneuver is also considered constant. However, as we track all hypotheses, it is still possible to estimate the correct maneuver if a driver changes his intention and therefore his behavior.

5) Action Model: an agent's action $\boldsymbol{a}=[a, \dot{\theta}]$ depends on its route and maneuver intentions, the kinematic states of all agents, and the map. It represents the third layer of the decision making process. Within this work, the same heuristics-based probabilistic action model $p(\boldsymbol{a} \mid r, m, X$, map $)$ is used as defined in [2]. The acceleration model is a non-linear mapping from features to a Gaussian distribution $p(a \mid r, m, X$, map $)=\mathcal{N}\left(\mu_{a}, \sigma_{a}^{2}\right)$, where $\mu_{a}$ is a function of $\left(r, m, X\right.$, map) and $\sigma_{a}$ is constant. The set of features is derived given the map and the kinematic state of all agents and describes the current context. The yaw rate is distributed according to the Gaussian $p(\dot{\theta} \mid r, \boldsymbol{x}, a$, map $)=$ $\mathcal{N}\left(\mu_{\dot{\theta}}, \sigma_{\dot{\theta}}^{2}\right)$, where $\mu_{\dot{\theta}}$ is a function of $(r, \boldsymbol{x}, a$, map $)$ so that the agent stays close to the center of its desired lane and $\sigma_{\dot{\theta}}$ is constant.

\section{B. Inference Algorithm}

Our goal is to estimate all drivers' route and maneuver intentions and to predict their future trajectories. The existence of different possibilities of routes and maneuvers generally leads to multi-modality in the predicted trajectories. As agents interact with each other and therefore their trajectories become interdependent, the discrete intentions of one agent may also lead to multi-modality in the prediction of another agent. Fig. 4 depicts a typical motion prediction situation illustrating this combinatorial aspect: depending on the intended route and maneuver of one agent, another agent may completely change its future behavior, resulting in different high probability clusters of the continuous state. As each UKF only represents a single Gaussian, this potential multi-modality creates the need for having multiple modes.

1) Multiple Models: given the route and maneuver of each agent, our evaluation suggests that the belief can reasonably be approximated by a single multivariate Gaussian. Thus, we define one UKF per possible combination of route and maneuver intentions of all agents $(R, M)$. The number of possible combinations of $K$ agents defines the number of modes of the MM-UKF and is given by $J=\prod_{i=0}^{K}\left(\sum_{r \in \mathcal{R}^{i}} \sum_{m \in \mathcal{M}^{i} \mid r} 1\right)$. Hence, the belief of the DBN is tracked using a set of $J$ weighted 


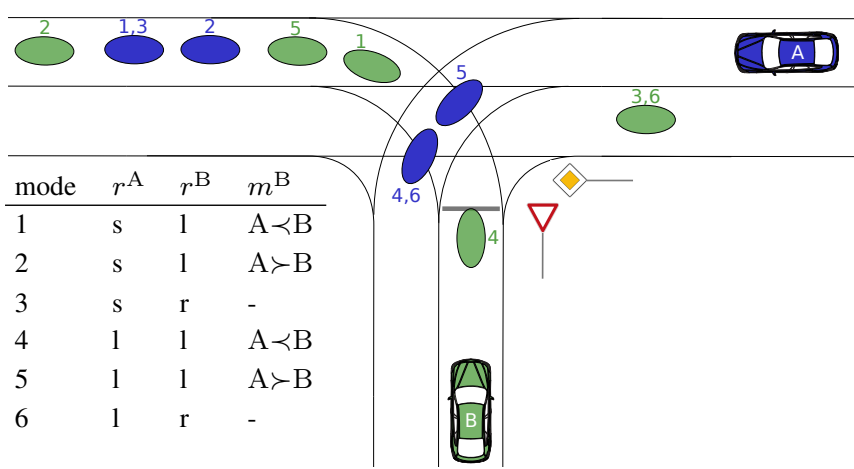

Fig. 4. Example of how different modes may result in different clusters of the continuous state (here shown as Gaussians of the positions) at a future time step, due to the interdependencies between agents. The routes are abbreviated as straight, left, and right.

UKFs $\mathcal{U}=\left\{\left(U^{1}, p\left(U^{1}\right)\right), \cdots,\left(U^{J}, p\left(U^{J}\right)\right)\right\}$. Each UKF $U^{j}=\left[X^{j}, R^{j}, M^{j}, A^{j}\right]^{\top}$ represents the complete scene including all agents and has attached the corresponding mode probability $p\left(U^{j}\right)$.

The general procedure is exemplarily depicted in Fig. 5: Initially, a multivariate Gaussian distribution of the kinematic state $X_{0}$ is derived from the first measurement $Z_{0}$ according to $p(X \mid Z)$ (step 1). Then, for each agent, the set of possible routes $\mathcal{R}_{0}$ and the set of possible maneuvers $\mathcal{M}_{0}$ for each of its possible routes is determined given its own state, the map, and all other agents' states (step 2-3). For each possible combination of $\left(R_{0}^{j}, M_{0}^{j}\right)$ of all agents, one UKF $U_{0}^{j}$ is created and initialized to the corresponding $R_{0}^{j}$ and $M_{0}^{j}$ and the Gaussian distribution $X_{0}^{j}=X_{0}$. Furthermore, it is assigned with the mode probability $p\left(R_{0}^{j}, M_{0}^{j}\right)=p\left(U_{0}^{j}\right)=$ $p\left(R_{0}^{j} \mid X_{0}^{j}\right.$, map $) p\left(M_{0}^{j} \mid R_{0}^{j}, X_{0}^{j}\right.$, map $)$.

For each UKF, the belief $U_{t}^{j}$ is predicted to $U_{t+1 \mid t}^{j}$ (step 4-5) and updated to $U_{t+1}^{j}$ (step 6), as shown in the next section. The probability of each mode is updated according to the measurement likelihood $\mathcal{L}\left(X_{t+1 \mid t}^{j} \mid Z_{t+1}\right)$ :

$$
p\left(U_{t+1}^{j}\right)=\frac{p\left(U_{t}^{j}\right) \mathcal{L}\left(X_{t+1 \mid t}^{j} \mid Z_{t+1}\right)}{\sum_{l \in J} p\left(U_{t}^{l}\right) \mathcal{L}\left(X_{t+1 \mid t}^{l} \mid Z_{t+1}\right)} .
$$

The Gaussian mixture that represents all modes, i.e., possible combinations of routes and maneuvers of all agents, is then given by the set of UKFs and corresponding probabilities.

As new agents appear in a new measurement or new routes or maneuvers emerge due to a route split or a new conflict area within the route horizon, the existing modes are duplicated accordingly in order to represent the new agents or the new possible intentions and the probabilities are split uniformly. If routes or maneuvers become impossible or agents disappear, the corresponding modes are removed or merged. Furthermore, unlikely modes can be disregarded to decrease runtime complexity (however, this option was disabled within the evaluation of this paper to ensure a fair comparison with the SMC-based inference). As the number of modes changes over time, the proposed method is of the type multiple model with variable structure [18]. To simplify the problem, the intentions of a driver are modeled

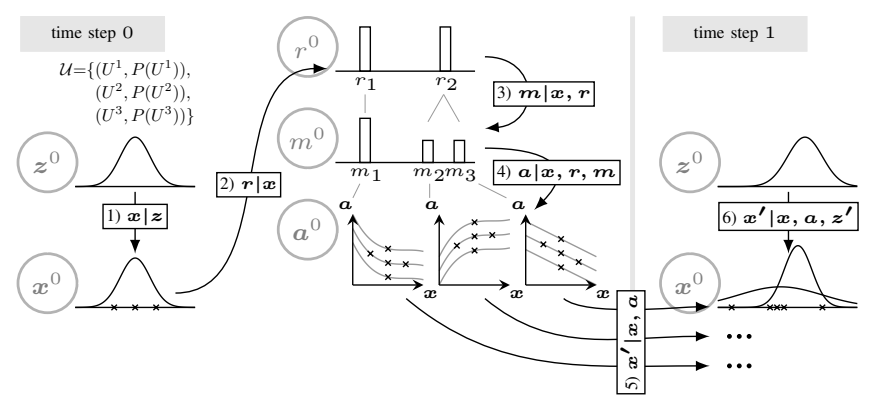

Fig. 5. MM-UKF inference exemplarily shown for a single vehicle with three different modes: $\left(r_{1}, m_{1}\right),\left(r_{2}, m_{2}\right),\left(r_{2}, m_{3}\right)$. Distributions are depicted simplified as being one dimensional. One UKF represents the complete state space, i.e., kinematic state, route, maneuver, and action of all agents in the scene. The steps of the algorithm are marked from 1 to 6 .

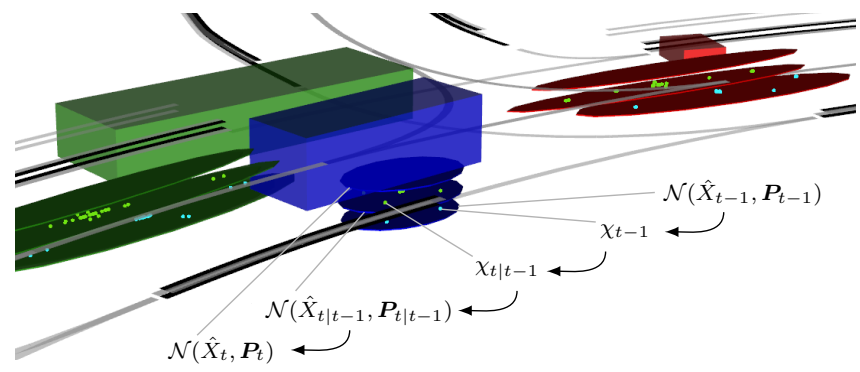

Fig. 6. Estimated belief and annotations for the Gaussians of the last posterior, the current prior and current posterior as well as the sigma points used for the prediction step. The posterior of the last time step and the prior of the current time step are drawn at lower height to improve visualization.

as constant over time, i.e., the probability of switching of the current mode is assumed to be zero. Thus, a non-interacting multiple model filter is applied. To model the possibility of mode switches, an interacting multiple model filter could be applied [19].

2) Single Model UKF: generally a UKF determines a deterministically chosen set of points that captures the mean and covariance of the original distribution and propagates them through the non-linear prediction and update functions. Then a new distribution is fitted to the resulting transformed points. As in our case the measurement is Gaussian and the measurement function is linear, sigma points are only utilized for the prediction step to be able to capture the non-linear system dynamics. A Gaussian is fitted directly after prediction and before the measurement update. Then a standard Kalman filter correction step is performed using the predicted Gaussian and the measurement. This procedure is depicted in Fig. 6 and will be explained in the remainder of this section. For the sake of brevity, the mode superscript $j$ is omitted within this section.

Typically, a set of $2 L+1$ sigma points is chosen, with $L$ being the dimensionality of the state, such that one sigma point is placed at the mean and the others are symmetrically spread around it. If the system is affected by nonadditive process noise, the mean state and the covariance must be augmented by one dimension per noise term, such that it can be accounted for by the sigma points. Additive noise can simply be added to the fitted Gaussian after the transformation. In this work, for each agent, the mean 
state and covariance are augmented by the non-additive process noise terms for acceleration and yaw-rate resulting in $\hat{\boldsymbol{x}}^{a, i}=\left[\begin{array}{lll}\hat{\boldsymbol{x}}^{i^{\top}} & \mu_{a}^{i} & \mu_{\dot{\theta}}^{i}\end{array}\right]^{\top}$ and $\boldsymbol{P}^{a, i}=\operatorname{diag}\left(\boldsymbol{P}^{i}, \sigma_{a}^{2}, \sigma_{\dot{\theta}}^{2}\right)$. where $\mu_{a}$ is a function of $\left(r, m, \hat{X}\right.$, map) and $\mu_{\dot{\theta}}$ a function of $(r, \hat{\boldsymbol{x}}, \hat{a}$, map) (step 4$)$. The overall augmented distribution consisting of all agents is thus given by a Gaussian with mean $\hat{X}^{a}=\left[\hat{\boldsymbol{x}}^{a, 1}, \cdots, \hat{\boldsymbol{x}}^{a, K}\right]^{\top}$ and covariance $\boldsymbol{P}^{a}=\operatorname{diag}\left(\boldsymbol{P}^{a, 1}, \cdots, \boldsymbol{P}^{a, K}\right)$.

For $K$ agents, the state has a size of $L=K(4+2)$, resulting in a total of $2 L+1=12 K+1$ sigma points being created. The sigma points are given by

$$
\begin{array}{ll}
\chi_{t}^{0} & =\hat{X}_{t}^{a} \\
\chi_{t}^{i} & =\hat{X}_{t}^{a}+\left(\sqrt{(L+\lambda) \boldsymbol{P}_{t}^{a}}\right)_{i}, \quad i=1, \ldots, L \\
\chi_{t}^{i} & =\hat{X}_{t}^{a}-\left(\sqrt{(L+\lambda) \boldsymbol{P}_{t}^{a}}\right)_{i}, \quad i=L+1, \ldots, 2 L,
\end{array}
$$

with $\left(\sqrt{(L+\lambda) \boldsymbol{P}_{t}^{a}}\right)_{i}$ being the $i$ th column of the matrix square root of $(L+\lambda) \boldsymbol{P}_{t}^{a}$. Each sigma point $\chi_{t}^{i}$ is then propagated through the transition function of the kinematic state (1) to the new time step (step 5). The resulting weighted sigma points $\chi_{t+1 \mid t}^{i}$ are then used to derive the predicted Gaussian with mean and covariance

$$
\begin{aligned}
& \hat{X}_{t+1 \mid t}=\sum_{i=0}^{2 L} W_{s}^{i} \chi_{t+1 \mid t}^{i} \\
& \boldsymbol{P}_{t+1 \mid t}=\boldsymbol{Q}+\sum_{i=0}^{2 L} W_{c}^{i}\left[\chi_{t+1 \mid t}^{i}-\hat{X}_{t+1 \mid t}\right]\left[\chi_{t+1 \mid t}^{i}-\hat{X}_{t+1 \mid t}\right]^{\top}
\end{aligned}
$$

with the state and covariance weights

$$
\begin{aligned}
& W_{s}^{0}=\frac{\lambda}{L+\lambda}, \quad W_{c}^{0}=W_{s}^{0}+\left(1-\alpha^{2}+\beta\right), \\
& W_{s}^{i}=W_{c}^{i}=\frac{1}{2(L+\lambda)}, \quad i=1, \ldots, 2 L
\end{aligned}
$$

and $\lambda=\alpha^{2}\left(L+\kappa^{\prime}\right)-L$. The choice of how to spread sigma points is extensively discussed in [17]. We found we were able to achieve good results when choosing what the author refers to as the Gauss set which uses the parameters $\alpha=1, \beta=0, \kappa^{\prime}=3-L$. The measurement step is then performed according to the standard Kalman filter (step 6 ), correcting the mean and covariance of the belief and allowing the determination of the measurement likelihood $\mathcal{L}\left(X_{t+1 \mid t}^{j} \mid Z_{t+1}\right)$ of the specific mode.

For the intention estimation process, the DBN is thus applied as a filter, comparing the different model hypotheses to the actual observations. As DBNs are generative models, i.e., they can generate values of any of their random variables, it is further possible to do a probabilistic forward simulation by iteratively predicting the current belief (including the estimated intentions) into subsequent time steps, applying the same models as for the filtering. Thus, for each UKF, one multi-agent trajectory is generated and weighted with the corresponding probability $p\left(U_{t}^{j}\right)$.

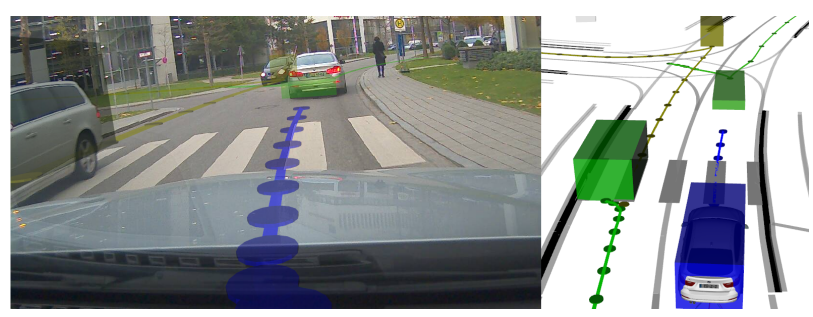

Fig. 7. Forward simulation of belief to generate multi-agent trajectories.

\section{Evaluation}

To highlight the differences of SMC-based and MM-UKFbased inference, their route and maneuver intention estimates are compared in simulation and real world scenarios. The data is recorded with a measuring vehicle using GPS/INS, lidar and radar sensors to detect and track surrounding traffic participants. We use a standard sequential importance resample (SIR) particle filter, also known as the bootstrap filter, with low-variance resampling. As SMC inference converges to the optimal estimate for $n \rightarrow \infty$, we compare the result of each estimator to the mean estimate over ten runs of SMC with a high number of samples $\left(n=10^{5}\right)$, which we abbreviate as BE for best estimator. The imprecision of each estimator is measured using the Kullback-Leibler divergence

$$
D_{\mathrm{KL}}\left(r_{\mathrm{BE}}^{i} \| r^{i}\right)=\sum_{j=1}^{|R|} r_{\mathrm{BE}, j}^{i} \log \frac{r_{\mathrm{BE}, j}^{i}}{p\left(r_{j}^{i}\right)}
$$

between the estimate $r^{i}=\left[p\left(r_{1}^{i}\right), \cdots, p\left(r_{|R|}^{i}\right)\right]$ and the estimate of the best estimator $r_{\mathrm{BE}}^{i}$ (analogous for maneuver estimates).

For each possible combination of routes and maneuvers of all agents, one forward simulation resulting in a multiagent trajectory is executed, as depicted in Fig. 7. Each forward simulation is initialized to the mean kinematic state of the given mode. As this procedure is the same for SMC-based and MM-UKF-based inference, the resulting trajectories given the same initial belief are identical. For a detailed evaluation of the forward simulation, we refer to [2].

A statistical evaluation of the mean $\bar{D}_{\mathrm{KL}}$ of all agents' route intentions over six different scenes (real and simulated) with 15 vehicles in total is depicted in Fig. 8. On average, the MM-UKF outperforms SMC with up to $n=50000$ particles, while having a maximum runtime per time step of $\tau=0.57 \mathrm{~s}$ compared to $\tau=7.9 \mathrm{~s}$ of SMC. As the estimation results are strongly dependent on the scenario, three different scenes are evaluated in more detail in Fig. 9. The first scene consists of three vehicles with human drivers approaching an intersection. The blue vehicle stops to yield to the green vehicle. As when turning right, the blue vehicle would not have to yield, it can be inferred that it either wants to turn left or go straight ahead $(t \approx 8 \mathrm{~s})$. As soon as the green vehicle has crossed the intersection, the blue vehicle starts to drive again and goes straight $(t \approx 9 \mathrm{~s})$. The MM-UKF has a runtime comparable to SMC with $n=600$ but outperforms SMC with up to $n=50000$. In the second scene, also using real data, the blue vehicle follows the green vehicle, 


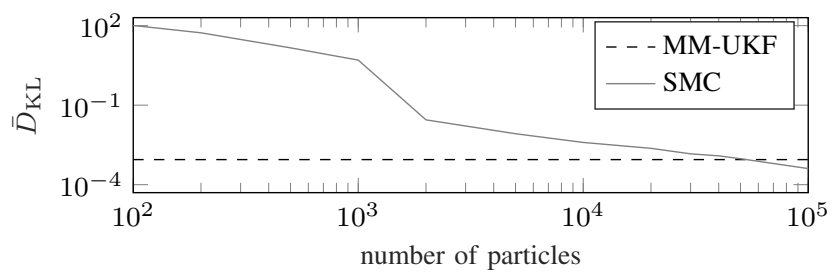

Fig. 8. Comparison of Kullback-Leibler divergence of MM-UKF and SMC with different number of particles over multiple scenes (15 agents in total).

which approaches the intersection and slows down before turning left. To maintain the desired headway distance, the blue vehicle also slows down, irrespective of its desired route $(t \approx 20 \mathrm{~s})$. As soon as the green vehicle has left the intersection, the blue vehicle accelerates again and continues straight $(t \approx 30 \mathrm{~s})$. The runtime of MM-UKF is similar to SMC with $n=300$, but it performs even better than SMC with $n=10^{5}$. The third scene shows how maneuvers and routes are estimated simultaneously. Initially, all three routes of the simulated blue vehicle have equal probability. As it only slows down slightly because of the upcoming curvature, but does not stop in order to yield to the green vehicle, it is inferred that neither going straight, nor yielding is likely. As soon as it starts turning, it is correctly inferred that it is turning left and merges before the green vehicle.

In all scenes, it was shown that SMC can result in high variances in the estimates depending on how many samples are used. As MM-UKF chooses its sigma points deterministically, multiple runs of the same scene always result in the same estimates. Although SMC is naturally able to achieve higher accuracy, this is usually at the cost of a higher runtime. In all of the evaluated examples, MM-UKF outperformed SMC in terms of accuracy per runtime. However, it has to be noted that the number of needed sigma points and thus the runtime of MM-UKF is dependent on the number of modes and vehicles in a scene. The results indicate that the presented MM-UKF-based inference provides a reasonable compromise between accuracy and runtime.

\section{CONCLUSIONS}

In this work, we propose a multiple model unscented Kalman filter (MM-UKF) based inference method for the intention estimation and trajectory prediction DBN presented in [2]. By including all agents within the state space, it is possible to account for interactions between multiple agents. Each possible combination of discrete route and maneuver intentions of all traffic participants forms one of the multiple modes of the filter. The comparison to SMC-based filtering suggests that the continuous belief given a specific mode can reasonably be approximated by a single multivariate Gaussian within the presented scenarios given the nonlinear transition models. It is shown that MM-UKF has a generally lower runtime than SMC with comparable accuracy or achieves improved accuracy given a similar runtime. The comparison of multiple runs with different SMC random initialization highlights the high variance in the estimation results, which is naturally not the case for MM-UKF. This ensures reproducible results allowing an easier validation of the overall framework. However, SMC has the advantage that the number of samples can be adjusted according to runtime requirements, whereas the number of sigma points is defined by the situation. Furthermore, discrete variables and non-additive noise terms can be added straightforwardly for SMC, whereas for MM-UKF the modes need to account for each discrete variable and the state of the sigma points needs to be augmented for each non-additive noise term.

Future work should focus on a statistical evaluation of an extensive dataset of urban traffic scenes. Detailed analysis of the scenes in which SMC achieves superior results will help in determining the approximation limits using Gaussian mixtures and sigma points in the current domain.

\section{REFERENCES}

[1] S. Lefèvre, D. Vasquez, and C. Laugier, "A survey on motion prediction and risk assessment for intelligent vehicles," Robomech J., vol. 1, no. 1, p. 1, 2014.

[2] J. Schulz, C. Hubmann, J. Löchner, and D. Burschka, "Interactionaware probabilistic behavior prediction in urban environments," in Int Conf. on Intell. Robots and Syst. (IROS) (accepted), IEEE, 2018.

[3] T. Li, S. Sun, T. P. Sattar, and J. M. Corchado, "Fight sample degeneracy and impoverishment in particle filters: A review of intelligent approaches," Expert Syst. with Appl., vol. 41, no. 8, pp. 3944-3954, 2014

[4] G. S. Aoude, V. R. Desaraju, L. H. Stephens, and J. P. How, "Behavior classification algorithms at intersections and validation using naturalistic data," in Intell. Veh. Symp. (IV), pp. 601-606, IEEE, 2011.

[5] M. Barbier, C. Laugier, O. Simonin, and J. Ibanez-Guzman, "Classification of Drivers Manoeuvre for Road Intersection Crossing with Synthetic and Real Data," in Intell. Veh. Symp. (IV), p. 7, IEEE, 2017.

[6] D. J. Phillips, T. A. Wheeler, and M. J. Kochenderfer, "Generalizable Intention Prediction of Human Drivers at Intersections," in Intell. Veh. Symp. (IV), pp. 1665-1670, IEEE, 2017.

[7] M. Liebner, M. Baumann, F. Klanner, and C. Stiller, "Driver intent inference at urban intersections using the intelligent driver model," in Intell. Veh. Symp. (IV), pp. 1162-1167, IEEE, 2012.

[8] Q. Tran and J. Firl, "Online maneuver recognition and multimodal trajectory prediction for intersection assistance using non-parametric regression," in Intell. Veh. Symp. (IV), pp. 918-923, IEEE, 2014.

[9] A. Armand, D. Filliat, and J. Ibanez-Guzman, "Modelling stop intersection approaches using gaussian processes," in Intell. Transp. Syst.(ITSC), pp. 1650-1655, IEEE, 2013.

[10] T. Gindele, S. Brechtel, and R. Dillmann, "Learning context sensitive behavior models from observations for predicting traffic situations," in Int. Conf. on Intell. Transp. Syst.-(ITSC), pp. 1764-1771, IEEE, 2013.

[11] D. Lenz, F. Diehl, M. T. Le, and A. Knoll, "Deep neural networks for Markovian interactive scene prediction in highway scenarios," in Intell. Veh. Symp. (IV), pp. 685-692, IEEE, 2017.

[12] J. Schulz, K. Hirsenkorn, J. Löchner, M. Werling, and D. Burschka, "Estimation of collective maneuvers through cooperative multi-agent planning," in Intell. Veh. Symp. (IV), pp. 624-631, IEEE, 2017.

[13] K. P. Murphy, "Dynamic bayesian networks," Probabilistic Graphical Models, M. Jordan, vol. 7, 2002

[14] S. J. Julier and J. K. Uhlmann, "Unscented filtering and nonlinear estimation," Proc. of the IEEE, vol. 92, no. 3, pp. 401-422, 2004.

[15] E. A. Wan and R. Van Der Merwe, "The unscented Kalman filter for nonlinear estimation," in Adaptive Syst. for Signal Process., Commun., and Control Symp., pp. 153-158, IEEE, 2000.

[16] M. N. Andersen, R. O. Andersen, and K. Wheeler, "Filtering in hybrid dynamic Bayesian networks," in Int. Conf. on Acoustics, Speech, and Signal Process., vol. 5, pp. V-773, IEEE, 2004.

[17] S. Bitzer, "The UKF exposed: How it works, when it works and when its better to sample.," doi:10.5281/zenodo.44386, 2016.

[18] X.-R. Li and Y. Bar-Shalom, "Multiple-Model Estimation with Variable Structure," IEEE Trans. on Automat. Control, vol. 41, no. 4, p. 16 , 1996

[19] Y. Bar-Shalom, P. K. Willett, and X. Tian, Tracking and data fusion. YBS publishing, 2011. 

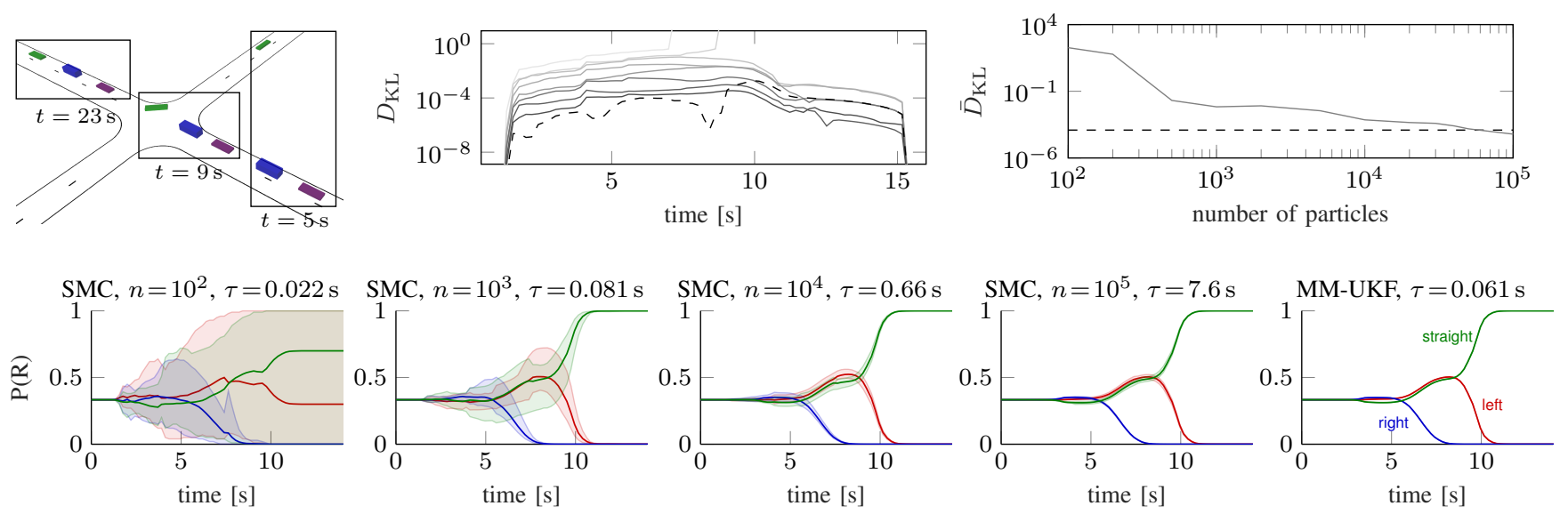

(a) Scene 1 (real data): The MM-UKF has a maximum runtime $\tau=0.061 \mathrm{~s}$, which is comparable to SMC with $n=600$, but performs as good as SMC with $n=50000$.
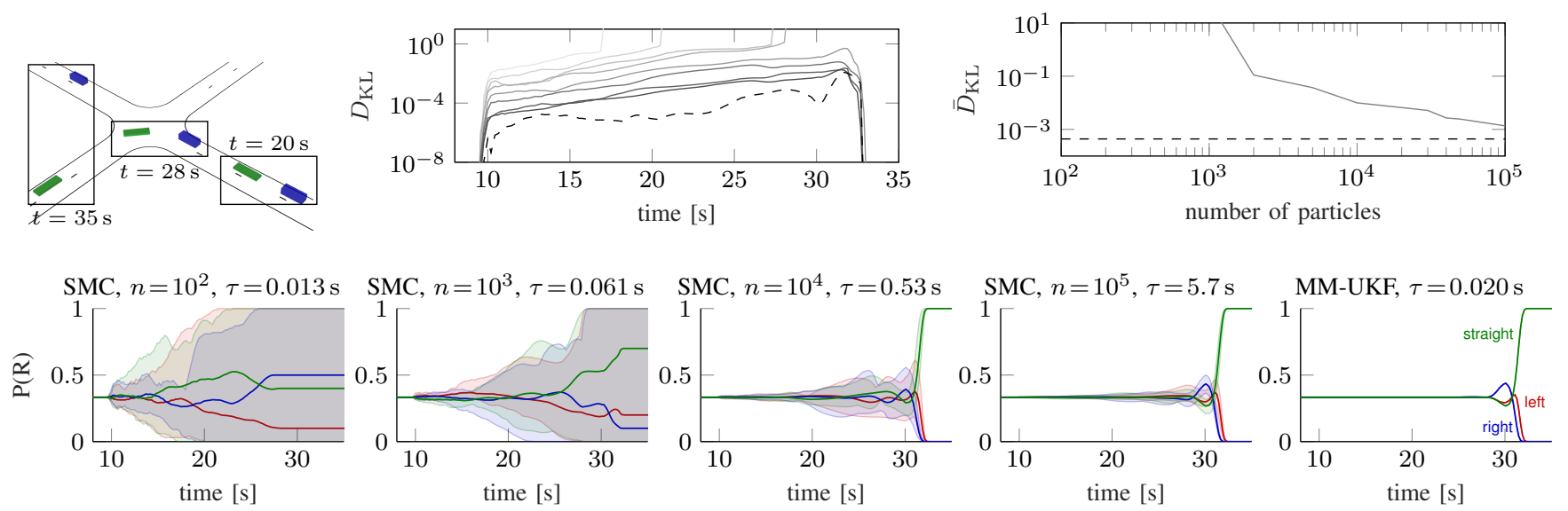

(b) Scene 2 (real data): The MM-UKF has a maximum runtime $\tau=0.020 \mathrm{~s}$, which is comparable to SMC with $n=300$, but performs even better than SMC with $n=100000$.
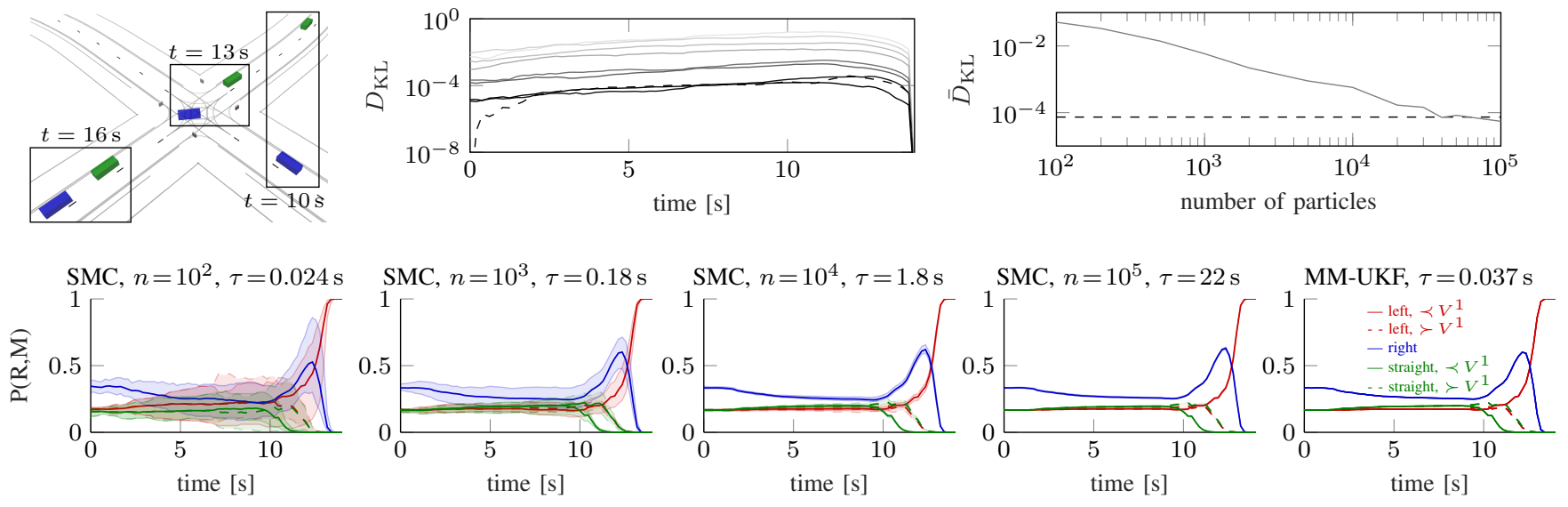

(c) Scene 3 (simulated data): The MM-UKF has a maximum runtime $\tau=0.037 \mathrm{~s}$, which is comparable to SMC with $n=200$, but performs as good as SMC with $n=40000$.

Fig. 9. Comparison of route/maneuver estimation accuracy and variance of MM-UKF and SMC with different number of particles. The plots depict the route/maneuver probabilities of the blue agent in each scene over ten runs with different random initialization (mean: thick line, min/max: filled areas). As MM-UKF has deterministic output, there is no variance in the estimate. Furthermore, the $D_{\mathrm{KL}}$ is plotted over time and its mean $\bar{D}_{\mathrm{KL}}$ over the number of used particles. The results of MM-UKF are depicted as dashed lines, whereas the ones of SMC as solid lines with different grayscale depending on the number of particles $(100,200,500,1000,5000,10000,50000,100000)$ : the more particles, the darker the line. The maximum runtime $\tau$ for one time step is based on non-optimized C++ code running on an Intel Core i7-5820K CPU @ 3.30GHz. 